\title{
Population Data on Finland 1900-20061
}

Updated by ANNELI MIETTINEN, M.Soc.Sc., Researcher

The Population Research Institute

Väestöliitto, the Family Federation of Finland

Population on 31 December

\begin{tabular}{llcccccccc}
\hline & Whole & \multicolumn{2}{c}{ Men } & \multicolumn{2}{c}{ Women } & \multicolumn{2}{c}{ Urban } & \multicolumn{2}{c}{$\begin{array}{c}\text { Rural } \\
\text { municipalities }\end{array}$} \\
& & 1000 & $\%$ & 1000 & $\%$ & 1000 & $\%$ & 1000 & $\%$ \\
\hline 1900 & 2655900 & 1311 & 49.3 & 1345 & 50.7 & 333 & 12.6 & 2323 & 87.4 \\
1910 & 2943400 & 1445 & 49.1 & 1499 & 50.9 & 432 & 14.7 & 2511 & 85.3 \\
1920 & 3147600 & 1533 & 48.7 & 1615 & 51.3 & 507 & 16.1 & 2640 & 83.9 \\
1930 & 3462700 & 1689 & 48.8 & 1774 & 51.2 & 715 & 20.6 & 2748 & 79.4 \\
1940 & 3695600 & 1793 & 48.5 & 1903 & 51.5 & 992 & 26.8 & 2704 & 73.2 \\
1950 & 4029800 & 1926 & 47.8 & 2104 & 52.2 & 1302 & 32.3 & 2727 & 67.7 \\
1960 & 4446200 & 2142 & 48.2 & 2304 & 51.8 & 1707 & 38.4 & 2739 & 61.6 \\
1970 & 4598300 & 2220 & 48.3 & 2378 & 51.7 & 2340 & 50.9 & 2258 & 49.1 \\
1980 & 4787800 & 2315 & 48.3 & 2473 & 51.7 & 2865 & 59.8 & 1923 & 40.2 \\
1990 & 4998500 & 2426 & 48.5 & 2572 & 51.5 & 3080 & 61.6 & 1919 & 38.4 \\
2000 & 5181100 & 2529 & 48.8 & 2652 & 51.2 & 3168 & 61.1 & 2013 & 38.9 \\
2005 & 5255600 & 2572 & 48.9 & 2683 & 51.1 & 3295 & 62.7 & 1961 & 37.3 \\
2006 & 5277000 & 2584 & 49.0 & 2693 & 51.0 & 3327 & 63.1 & 1950 & 36.9 \\
$2007^{*}$ & 5300000 & & & & & & & & \\
\hline
\end{tabular}

"The classification of municipalities into urban and rural areas changed in 1996. The division used here is the same as the one used by Statistics Finland.

* Statistics Finland, preliminary data

Age structure

\begin{tabular}{rrrrrrrrrr}
\hline & \multicolumn{3}{c}{ Whole population } & \multicolumn{3}{c}{ Men } & \multicolumn{3}{c}{ Women } \\
& $0-14$ & $15-64$ & $65-$ & $0-14$ & $15-64$ & $65-$ & $0-14$ & $15-64$ & $65-$ \\
\hline 1900 & 35.0 & 59.6 & 5.4 & 35.9 & 59.4 & 4.7 & 34.3 & 59.7 & 6.0 \\
1910 & 35.6 & 58.6 & 5.8 & 36.7 & 58.3 & 5.0 & 34.6 & 59.0 & 6.4 \\
1920 & 33.4 & 60.7 & 5.9 & 34.8 & 60.0 & 5.2 & 32.0 & 61.3 & 6.7 \\
1930 & 29.4 & 64.3 & 6.3 & 30.6 & 64.1 & 5.3 & 28.3 & 64.5 & 7.2 \\
1940 & 26.9 & 66.7 & 6.4 & 28.2 & 66.5 & 5.3 & 25.7 & 66.9 & 7.4 \\
1950 & 30.0 & 63.3 & 6.7 & 32.0 & 62.8 & 5.2 & 28.1 & 63.9 & 8.0 \\
1960 & 30.1 & 62.4 & 7.4 & 31.9 & 62.3 & 5.7 & 28.5 & 62.5 & 8.9 \\
1970 & 24.3 & 66.3 & 9.3 & 25.7 & 67.1 & 7.2 & 23.0 & 65.7 & 11.3 \\
1980 & 20.2 & 67.8 & 12.1 & 21.4 & 69.7 & 8.9 & 19.1 & 66.0 & 14.9 \\
1990 & 19.3 & 67.2 & 13.5 & 20.3 & 69.8 & 9.9 & 18.3 & 64.8 & 16.9 \\
2000 & 18.1 & 66.9 & 15.0 & 18.9 & 69.3 & 11.8 & 17.3 & 64.7 & 18.0 \\
2005 & 17.3 & 66.7 & 16.0 & 18.0 & 68.9 & 13.1 & 16.6 & 64.7 & 18.8 \\
2006 & 17.1 & 66.4 & 16.5 & 17.8 & 68.6 & 13.6 & 16.4 & 64.4 & 19.2 \\
\hline
\end{tabular}

${ }^{1}$ The data are based on the statistics of Statistics Finland, Helsinki. 
Age-specific fertility rate

\begin{tabular}{|c|c|c|c|c|c|c|c|c|}
\hline & \multicolumn{7}{|c|}{ Per 1,000 women } & \multirow{2}{*}{$\begin{array}{l}\text { Total fertility } \\
\text { per woman }\end{array}$} \\
\hline & $15-19$ & $20-24$ & $25-29$ & $30-34$ & $35-39$ & $40-44$ & $45-49$ & \\
\hline $1901-10$ & 16.8 & 147.7 & 226.8 & 227.4 & 192.6 & 109.1 & 16.2 & 4.68 \\
\hline $1911-20$ & 14.9 & 122.8 & 181.4 & 181.9 & 155.2 & 92.9 & 14.0 & 3.81 \\
\hline $1921-30$ & 14.2 & 110.0 & 156.9 & 143.8 & 116.8 & 67.4 & 10.5 & 3.10 \\
\hline $1931-40$ & 14.4 & 96.5 & 126.2 & 108.9 & 82.8 & 43.5 & 5.6 & 2.39 \\
\hline $1941-45$ & 11.3 & 105.2 & 144.9 & 124.3 & 88.4 & 41.8 & 5.4 & 2.60 \\
\hline $1946-50$ & 25.7 & 161.9 & 189.4 & 147.5 & 100.5 & 43.3 & 5.0 & 3.37 \\
\hline $1951-55$ & 27.2 & 157.6 & 165.8 & 125.0 & 81.0 & 35.1 & 3.9 & 2.98 \\
\hline $1956-60$ & 29.3 & 161.4 & 159.6 & 108.1 & 67.5 & 27.5 & 2.8 & 2.78 \\
\hline $1961-65$ & 30.7 & 156.7 & 156.0 & 98.8 & 55.7 & 22.5 & 2.2 & 2.61 \\
\hline $1966-70$ & 34.7 & 131.9 & 125.9 & 76.5 & 39.7 & 13.7 & 1.4 & 2.12 \\
\hline $1971-75$ & 27.9 & 104.3 & 106.6 & 55.8 & 23.3 & 6.1 & 0.5 & 1.62 \\
\hline $1976-80$ & 22.0 & 96.8 & 114.6 & 67.5 & 26.4 & 6.0 & 0.3 & 1.67 \\
\hline $1981-85$ & 15.7 & 85.0 & 122.8 & 75.3 & 32.1 & 6.7 & 0.4 & 1.69 \\
\hline $1986-90$ & 12.3 & 70.2 & 126.0 & 85.6 & 33.2 & 7.5 & 0.4 & 1.68 \\
\hline $1991-95$ & 10.9 & 71.0 & 134.2 & 101.4 & 39.2 & 7.7 & 0.4 & 1.82 \\
\hline $1996-00$ & 9.5 & 61.4 & 119.6 & 101.6 & 44.7 & 8.8 & 0.5 & 1.73 \\
\hline 2001 & 10.6 & 59.7 & 114.1 & 101.9 & 47.5 & 9.7 & 0.5 & 1.73 \\
\hline 2002 & 11.2 & 57.2 & 112.5 & 102.9 & 47.9 & 9.8 & 0.6 & 1.72 \\
\hline 2003 & 10.3 & 57.0 & 115.5 & 106.9 & 49.4 & 10.8 & 0.5 & 1.76 \\
\hline 2004 & 10.6 & 58.0 & 116.1 & 111.5 & 51.1 & 11.0 & 0.5 & 1.80 \\
\hline 2005 & 10.3 & 57.4 & 116.3 & 112.9 & 51.5 & 10.7 & 0.6 & 1.80 \\
\hline 2006 & 9.4 & 58.4 & 116.8 & 117.9 & 53.0 & 11.0 & 0.5 & 1.84 \\
\hline
\end{tabular}

Gross and net reproduction rate

\begin{tabular}{lccccc}
\hline & $\begin{array}{c}\text { Gross } \\
\text { reproduction } \\
\text { rate }\end{array}$ & $\begin{array}{c}\text { Net } \\
\text { reproduction } \\
\text { rate }\end{array}$ & .. & $\begin{array}{c}\text { Gross } \\
\text { reproduction } \\
\text { rate }\end{array}$ & $\begin{array}{c}\text { Net } \\
\text { reproduction } \\
\text { rate }\end{array}$ \\
\hline $1901-10$ & 2.278 &.. & $1966-70$ & 1.035 & 1.009 \\
$1911-20$ & 1.849 &.. & $1971-75$ & 0.790 & 0.773 \\
$1921-30$ & 1.504 & 0.958 & $1981-85$ & 0.826 & 0.801 \\
$1931-35$ & 1.167 & 0.962 & $1986-90$ & 0.819 & 0.815 \\
$1936-40$ & 1.162 & 1.053 & $1991-95$ & 0.894 & 0.808 \\
$1941-45$ & 1.257 & 1.382 & $1996-00$ & 0.847 & 0.882 \\
$1946-50$ & 1.622 & 1.373 & $2001-05$ & 0.861 & 0.837 \\
$1951-55$ & 1.452 & 1.301 & 2006 & 0.900 & 0.852 \\
$1956-60$ & 1.357 & 1.236 & & & 0.893 \\
$1961-65$ & 1.276 & & & & \\
\hline
\end{tabular}


Median age of mother at first birth

\begin{tabular}{lrrr}
\hline & Median age & Median age \\
\hline $1961-65$ & 23.3 & $1996-00$ & 27.5 \\
$1966-70$ & 22.7 & 2001 & 27.2 \\
$1971-75$ & 24.0 & 2002 & 27.4 \\
$1976-80$ & 25.2 & 2003 & 27.6 \\
$1981-85$ & 25.4 & 2004 & 27.7 \\
$1986-90$ & 26.3 & 2005 & 27.8 \\
$1991-95$ & 27.0 & 2006 & 27.8 \\
\hline
\end{tabular}

Live births

\begin{tabular}{lccrcc}
\hline & $\begin{array}{c}\text { Annual mean/ } \\
\text { Number }\end{array}$ & $\begin{array}{c}\text { Per 1,000 of } \\
\text { mean population }\end{array}$ & & $\begin{array}{c}\text { Annual mean/ } \\
\text { Number }\end{array}$ & $\begin{array}{c}\text { Per 1,000 of } \\
\text { mean population }\end{array}$ \\
\hline $1901-10$ & 90292 & 32.4 & $1986-90$ & 62534 & 12.6 \\
$1911-20$ & 83045 & 27.0 & $1991-95$ & 65050 & 12.8 \\
$1921-30$ & 78184 & 23.6 & $1996-00$ & 58295 & 11.3 \\
$1931-40$ & 70584 & 19.7 & 2001 & 56189 & 10.8 \\
$1941-50$ & 92614 & 24.2 & 2002 & 55555 & 10.7 \\
$1951-60$ & 88024 & 20.8 & 2003 & 56630 & 10.9 \\
$1961-70$ & 76466 & 16.8 & 2004 & 57758 & 11.0 \\
$1971-80$ & 62789 & 13.3 & 2005 & 57745 & 11.0 \\
$1981-85$ & 64868 & 13.4 & 2006 & 58840 & 11.2 \\
\hline
\end{tabular}

Illegitimate live births

\begin{tabular}{lccrcc}
\hline & $\begin{array}{c}\text { Annual mean/ } \\
\text { Number }\end{array}$ & $\begin{array}{c}\text { Percent of } \\
\text { live births }\end{array}$ & & $\begin{array}{c}\text { Annual mean/ } \\
\text { Number }\end{array}$ & $\begin{array}{c}\text { Percent of } \\
\text { live births }\end{array}$ \\
\hline $1901-10$ & 6120 & 6.8 & $1986-90$ & 13301 & 21.3 \\
$1911-20$ & 6573 & 6.8 & $1991-95$ & 19628 & 30.2 \\
$1921-30$ & 6606 & 8.4 & $1996-00$ & 21781 & 37.4 \\
$1931-40$ & 5349 & 7.6 & 2001 & 22222 & 39.5 \\
$1941-50$ & 5629 & 6.1 & 2002 & 22156 & 39.9 \\
$1951-60$ & 3819 & 4.3 & 2003 & 22649 & 40.0 \\
$1961-70$ & 3621 & 4.7 & 2004 & 23554 & 40.8 \\
$1971-80$ & 6182 & 9.8 & 2005 & 23319 & 40.4 \\
$1981-85$ & 9388 & 14.5 & 2006 & 23858 & 40.5 \\
\hline
\end{tabular}

Abortions

\begin{tabular}{lccccc}
\hline & $\begin{array}{c}\text { Annual mean/ } \\
\text { Number }\end{array}$ & $\begin{array}{c}\text { Percent of } \\
\text { live births }\end{array}$ & & $\begin{array}{c}\text { Annual mean/ } \\
\text { Number }\end{array}$ & $\begin{array}{c}\text { Percent of } \\
\text { live births }\end{array}$ \\
\hline 1970 & 14757 & 22.9 & 2001 & 10738 & 19.1 \\
$1971-75$ & 22105 & 36.3 & 2002 & 10974 & 19.8 \\
$1976-80$ & 17081 & 26.4 & 2003 & 10767 & 19.0 \\
$1981-85$ & 13764 & 21.2 & 2004 & 11162 & 19.0 \\
$1986-90$ & 12791 & 20.5 & 2005 & 10969 & 19.3 \\
$1991-95$ & 10610 & 16.3 & 2006 & 10645 & 18.1 \\
$1996-00$ & 10638 & 18.3 & & & \\
\hline
\end{tabular}


Marriages

\begin{tabular}{|c|c|c|c|c|c|c|c|}
\hline & $\begin{array}{l}\text { Annual mea } \\
\text { Marriages }\end{array}$ & $\begin{array}{l}\text { / Number } \\
\text { Registered } \\
\text { unions }^{1} \\
\end{array}$ & $\begin{array}{c}\text { Per } 1,000 \\
\text { of mean } \\
\text { population }\end{array}$ & & $\begin{array}{l}\text { Annual me } \\
\text { Marriages }\end{array}$ & $\begin{array}{c}\text { Ran/ Number } \\
\text { Registered } \\
\text { unions }\end{array}$ & $\begin{array}{l}\text { Per } 1,000 \\
\text { of mean } \\
\text { population }\end{array}$ \\
\hline $\begin{array}{l}1901-10 \\
1911-20\end{array}$ & $\begin{array}{l}18947 \\
18922\end{array}$ & $\begin{array}{l}. . \\
. .\end{array}$ & $\begin{array}{l}6.8 \\
6.2\end{array}$ & $\begin{array}{l}1986-90 \\
1991-95\end{array}$ & $\begin{array}{l}25516 \\
24317\end{array}$ & $\begin{array}{l}. . \\
. .\end{array}$ & $\begin{array}{l}5.2 \\
4.8\end{array}$ \\
\hline $1921-30$ & 23725 & .. & 7.2 & 1996-00 & 24470 & .. & 4.7 \\
\hline $1931-40$ & 28491 & .. & 7.9 & 2001 & 24830 & .. & 4.8 \\
\hline $1941-50$ & 37367 & .. & 9.8 & $2002^{*}$ & 26969 & 446 & 5.2 \\
\hline $1951-60$ & 32191 & .. & 7.6 & 2003 & 25815 & 190 & 5.0 \\
\hline $1961-70$ & 37398 & .. & 8.2 & 2004 & 29342 & 186 & 5.6 \\
\hline $1971-80$ & 32575 & .. & 7.0 & 2005 & 29283 & 200 & 5.6 \\
\hline $1981-85$ & 28867 & .. & 5.9 & 2006 & 28236 & 191 & 5.4 \\
\hline
\end{tabular}

"Since 2002, same-sex couples have been able to register their union.

Median age at first marriage

\begin{tabular}{lccrcc}
\hline & Men & Women & & Men & Women \\
\hline $1901-05$ & 26.1 & 23.5 & $1966-70$ & 23.6 & 22.2 \\
$1906-10$ & 26.2 & 23.6 & $1971-75$ & 24.0 & 22.7 \\
$1911-15$ & 26.4 & 23.7 & $1976-80$ & 25.2 & 23.3 \\
$1916-20$ & 27.0 & 24.0 & $1981-85$ & 26.2 & 24.2 \\
$1921-25$ & 26.9 & 24.0 & $1986-90$ & 27.1 & 25.2 \\
$1926-30$ & 26.6 & 23.9 & $1991-95$ & 28.1 & 26.3 \\
$1931-35$ & 27.1 & 24.4 & $1996-00$ & 29.3 & 27.1 \\
$1936-40$ & 27.6 & 24.7 & 2001 & 29.6 & 27.3 \\
$1941-45$ & 27.6 & 24.3 & 2002 & 29.8 & 27.7 \\
$1946-50$ & 26.1 & 23.7 & 2003 & 29.9 & 27.8 \\
$1951-55$ & 24.9 & 23.2 & 2004 & 30.4 & 28.2 \\
$1956-60$ & 24.6 & 22.8 & 2005 & 30.2 & 28.2 \\
$1961-65$ & 24.1 & 22.3 & 2006 & 30.5 & 28.3 \\
\hline
\end{tabular}

\section{Consensual unions}

Persons living in consensual union of all persons living in union $(\%)^{*}$

\begin{tabular}{lccccccc} 
& $15-24$ & $25-29$ & $30-34$ & $35-44$ & $45-64$ & $15-64$ & $\begin{array}{c}\text { consensual union } \\
1981\end{array}$ \\
\hline 44.6 & 19.4 & 12.4 & 6.5 & 2.5 & 10.3 & 214000 \\
1985 & 55.0 & 27.1 & 13.5 & 7.4 & 4.4 & 12.8 & 283000 \\
1990 & 69.7 & 39.3 & 21.6 & 12.4 & 6.8 & 21.3 & 366580 \\
1995 & 77.4 & 47.2 & 28.9 & 17.3 & 8.8 & 21.0 & 430444 \\
2000 & 81.8 & 54.6 & 34.8 & 22.7 & 11.4 & 24.8 & 509456 \\
2005 & 83.6 & 57.6 & 36.8 & 25.8 & 14.0 & 27.4 & 564799 \\
2006 & 83.9 & 58.5 & 37.3 & 26.1 & 14.6 & 28.0 & 598806 \\
\hline
\end{tabular}

"Until 1990, estimation by Statistics Finland on the basis of information from Labor Force Surveys. Since 1990, consensual unions are derived from population registers. 
Divorces

\begin{tabular}{lccccc}
\hline & $\begin{array}{c}\text { Annual mean/ } \\
\text { Number }\end{array}$ & $\begin{array}{c}\text { Per 1,000 } \\
\text { of mean } \\
\text { population }\end{array}$ & & $\begin{array}{c}\text { Annual mean/ } \\
\text { Number }\end{array}$ & $\begin{array}{c}\text { Per 1,000 } \\
\text { of mean } \\
\text { population }\end{array}$ \\
\hline $1931-40$ & 1391 & 0.4 & $1996-00$ & 13819 & 2.7 \\
$1941-50$ & 3721 & 1.0 & 2001 & 13589 & 2.6 \\
$1951-60$ & 3584 & 0.8 & $2002^{*}$ & 13360 & 2.6 \\
$1961-70$ & 4860 & 1.1 & 2003 & 13475 & 2.6 \\
$1971-80$ & 9389 & 2.0 & 2004 & 13234 & 2.5 \\
$1981-85$ & 9538 & 2.0 & 2005 & 13383 & 2.6 \\
$1986-90$ & 11898 & 2.4 & 2006 & 13255 & 2.5 \\
$1991-95$ & 13260 & 2.6 & & & \\
\hline
\end{tabular}

"Since 2002, divorce from a registered same-sex couple is included in the data.

Families with children under 18 years of age by type of family (\%)

\begin{tabular}{lcccccc}
\hline & $\begin{array}{c}\text { Married } \\
\text { couples with } \\
\text { children }^{1}\end{array}$ & $\begin{array}{c}\text { Couples living } \\
\text { in consensual } \\
\text { union with } \\
\text { children }^{2}\end{array}$ & $\begin{array}{c}\text { Mothers with } \\
\text { children }\end{array}$ & $\begin{array}{c}\text { Fathers with } \\
\text { children }\end{array}$ & $\begin{array}{c}\text { Registered } \\
\text { couples with } \\
\text { children }\end{array}$ & Number \\
\hline 1950 & 85.9 &.. & 12.4 & 1.7 &.. & 599329 \\
1960 & 88.7 &.. & 9.9 & 1.3 &.. & 678046 \\
1970 & 88.9 & 0.9 & 9.0 & 1.2 &.. & 677035 \\
1980 & 83.1 & 4.7 & 10.9 & 1.4 &.. & 688732 \\
1990 & 76.6 & 9.4 & 12.3 & 1.7 &.. & 640637 \\
1995 & 70.2 & 12.3 & 15.4 & 2.1 &.. & 639610 \\
2000 & 65.1 & 15.5 & 17.0 & 2.4 &.. & 612627 \\
2005 & 62.3 & 17.7 & 17.4 & 2.5 & 0.0 & 591528 \\
2006 & 62.0 & 18.1 & 17.4 & 2.6 & 0.0 & 589448 \\
\hline
\end{tabular}

${ }^{1}$ Includes not-married couples with children under 18 years, until 1970.

${ }^{2}$ Consensual unions are estimated on the basis of population survey (1970) and on population registers (since 1980). 
Families with children under 18 years of age by type of family and number of children (\%)

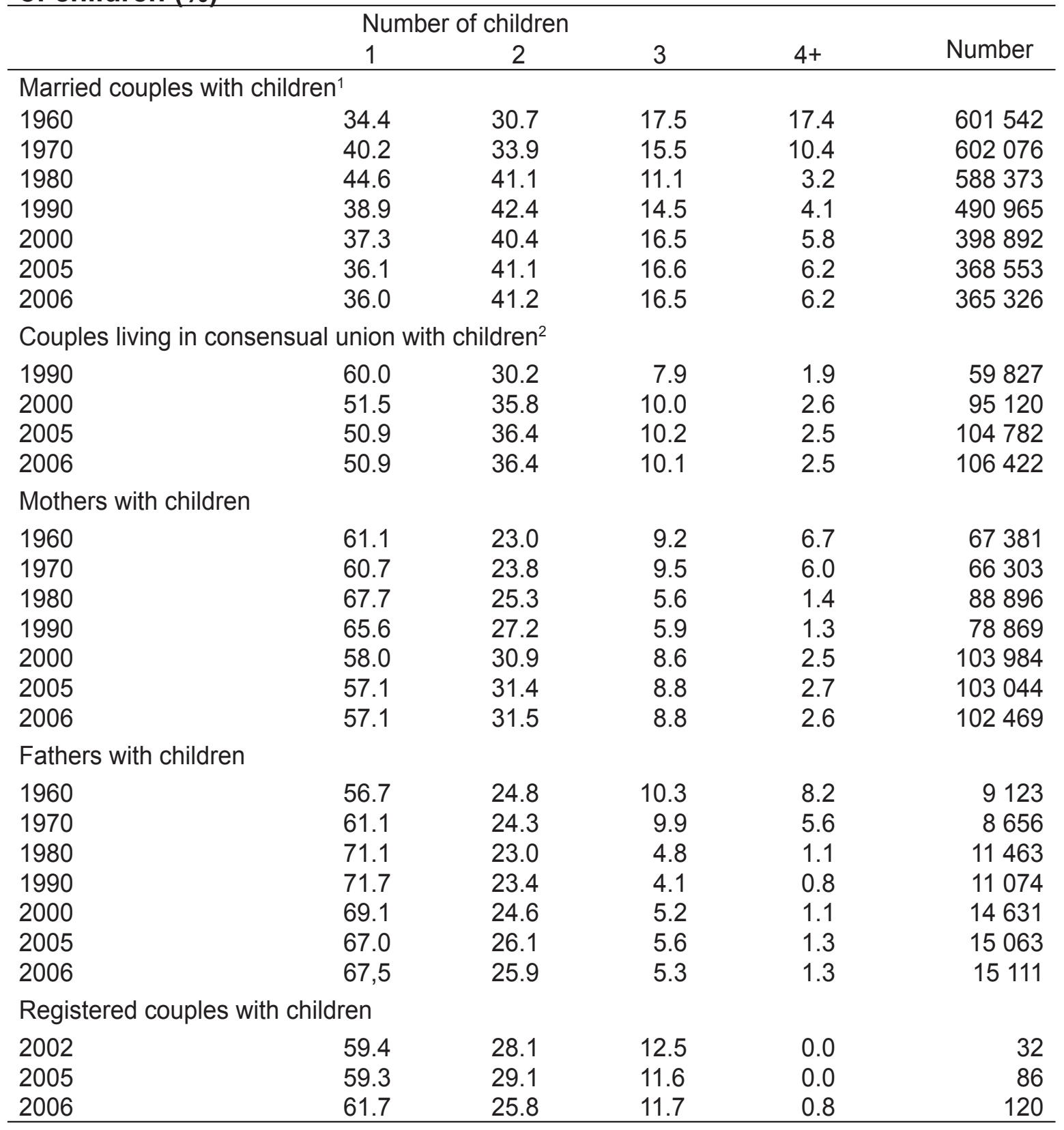

${ }^{1}$ Includes not-married couples with children under 18 years, until 1970.

${ }^{2}$ Consensual unions are estimated on the basis of population registers (since 1980). 
Deaths

\begin{tabular}{lccccccc}
\hline & $\begin{array}{c}\text { Annual } \\
\text { mean/ } \\
\text { number }\end{array}$ & $\begin{array}{c}\text { Per 1,000 } \\
\text { of mean } \\
\text { population }\end{array}$ & $\begin{array}{c}\text { Infant } \\
\text { mortality }\end{array}$ & & $\begin{array}{c}\text { Annual } \\
\text { mean/ } \\
\text { number }\end{array}$ & $\begin{array}{c}\text { Per 1,000 } \\
\text { of mean } \\
\text { population }\end{array}$ & $\begin{array}{c}\text { Infant } \\
\text { mortality }\end{array}$ \\
\hline $1901-10$ & 52099 & 18.7 & 123.8 & $1986-90$ & 48663 & 9.8 & 5.9 \\
$1911-20$ & 58284 & 19.0 & 111.8 & $1991-95$ & 49481 & 9.8 & 4.8 \\
$1921-30$ & 49573 & 14.9 & 91.8 & $1996-00$ & 49244 & 9.6 & 3.9 \\
$1931-40$ & 50230 & 14.0 & 72.1 & 2001 & 48550 & 9.4 & 3.2 \\
$1941-50$ & 51838 & 13.6 & 56.6 & 2002 & 49418 & 9.5 & 3.0 \\
$1951-60$ & 39381 & 9.3 & 28.4 & 2003 & 48996 & 9.4 & 3.1 \\
$1961-70$ & 43494 & 9.5 & 16.6 & 2004 & 47600 & 9.1 & 3.3 \\
$1971-80$ & 44243 & 9.4 & 9.7 & 2005 & 47928 & 9.1 & 3.0 \\
$1981-85$ & 45299 & 9.3 & 6.3 & 2006 & 48065 & 9.1 & 2.8 \\
\hline
\end{tabular}

"Mortality during the first year of life per 1000 live births.

Life expectancy

\begin{tabular}{llllll}
\hline & Men & Women & & Men & Women \\
\hline $1911-20^{1}$ & 43.4 & 49.1 & $1981-85$ & 70.1 & 78.4 \\
$1921-30$ & 50.7 & 55.1 & $1986-90$ & 70.7 & 78.8 \\
$1931-40^{2}$ & 54.5 & 59.6 & $1991-95$ & 72.1 & 79.7 \\
$1941-45^{2}$ & 54.6 & 61.1 & $1996-00$ & 73.5 & 80.8 \\
$1946-50$ & 58.6 & 65.8 & 2001 & 74.6 & 81.5 \\
$1951-55$ & 63.4 & 69.8 & 2002 & 74.9 & 81.5 \\
$1956-60$ & 65.9 & 71.6 & 2003 & 75.1 & 81.8 \\
$1961-65$ & 65.4 & 72.6 & 2004 & 75.3 & 82.3 \\
$1966-70$ & 65.9 & 73.6 & 2005 & 75.5 & 82.3 \\
$1971-75$ & 66.7 & 75.2 & 2006 & 75.8 & 82.8 \\
$1976-80$ & 68.5 & 77.2 & & & \\
\hline
\end{tabular}

${ }^{1}$ Including deaths due to war operations

${ }^{2}$ Excluding deaths due to war operations

\section{Excess of births over deaths}

\begin{tabular}{lccccc}
\hline & $\begin{array}{c}\text { Annual } \\
\text { mean/ } \\
\text { number }\end{array}$ & $\begin{array}{c}\text { Per } 1,000 \\
\text { of mean } \\
\text { population }\end{array}$ & & $\begin{array}{c}\text { Annual } \\
\text { mean/ } \\
\text { number }\end{array}$ & $\begin{array}{c}\text { Per } 1,000 \\
\text { of mean } \\
\text { population }\end{array}$ \\
\hline $1901-10$ & 38194 & 13.7 & $1986-90$ & 13871 & 2.8 \\
$1911-20$ & 24760 & 8.1 & $1991-95$ & 15569 & 3.1 \\
$1921-30$ & 28611 & 8.7 & $1996-00$ & 9051 & 1.8 \\
$1931-40$ & 20355 & 5.7 & 2001 & 7639 & 1.5 \\
$1941-50$ & 40776 & 10.6 & 2002 & 6137 & 1.2 \\
$1951-60$ & 48643 & 11.5 & 2003 & 7634 & 1.5 \\
$1961-70$ & 32972 & 7.2 & 2004 & 10158 & 1.9 \\
$1971-80$ & 18546 & 3.9 & 2005 & 9817 & 1.9 \\
$1981-85$ & 19569 & 4.0 & 2006 & 10775 & 2.0 \\
\hline
\end{tabular}


International migration

\begin{tabular}{lcc}
\hline & $\begin{array}{c}\text { Emigrants who have } \\
\text { obtained a passport }\end{array}$ & $\begin{array}{c}\text { Per } 1,000 \text { of } \\
\text { mean population }\end{array}$ \\
\hline $1901-10$ & 150832 & 5.4 \\
$1911-20$ & 67346 & 2.2 \\
$1921-30$ & 58559 & 1.8 \\
$1931-40$ & 8844 & 0.2 \\
\hline & \multicolumn{2}{c}{ Net migration } \\
& Annual mean/Number & Per 1,000 of \\
\hline $1941-50$ & -4100 & mean population \\
$1951-60$ & -7003 & -1.1 \\
$1961-70$ & -17763 & -1.5 \\
$1971-75$ & 1936 & -3.9 \\
$1976-80$ & -7493 & 0.4 \\
$1981-85$ & 5347 & -1.6 \\
$1986-90$ & 2905 & 1.1 \\
$1991-95$ & 7222 & 0.6 \\
$1996-00$ & 3031 & 1.4 \\
2001 & 5802 & 0.6 \\
2002 & 5222 & 1.1 \\
2003 & 5755 & 1.0 \\
2004 & 6677 & 1.1 \\
2005 & 8986 & 1.3 \\
2006 & 10344 & 1.7 \\
\hline
\end{tabular}

Internal migration

\begin{tabular}{cccccc}
\hline & $\begin{array}{c}\text { Annual mean/ } \\
\text { Number }\end{array}$ & $\begin{array}{c}\text { Percent of } \\
\text { mean popula- } \\
\text { tion }\end{array}$ & & $\begin{array}{c}\text { Annual mean/ } \\
\text { Number }\end{array}$ & $\begin{array}{c}\text { Percent of mean } \\
\text { population }\end{array}$ \\
\hline $1901-10$ & 74784 & 2.7 & $1986-90$ & 200737 & 4.1 \\
$1911-20$ & 85111 & 2.8 & $1991-95$ & 189999 & 3.8 \\
$1921-30$ & 79248 & 2.4 & $1996-00$ & 247151 & 4.8 \\
$1931-39$ & 136470 & 3.8 & 2001 & 280140 & 5.4 \\
$1941-50$ & 132778 & 3.5 & 2002 & 272023 & 5.2 \\
$1951-60$ & 172098 & 4.0 & 2003 & 271333 & 5.2 \\
$1961-70$ & 219330 & 4.8 & 2004 & 281976 & 5.4 \\
$1971-80$ & 213569 & 4.6 & 2005 & 290149 & 5.5 \\
$1981-85$ & 193681 & 4.0 & 2006 & 286522 & 5.4 \\
\hline
\end{tabular}

*Average in-migration to rural and urban municipalities. 
Foreign citizens in Finland

\begin{tabular}{rcclr}
\hline & $\begin{array}{c}\text { Number of } \\
\text { foreigners }\end{array}$ & $\begin{array}{c}\text { Percent of } \\
\text { total population }\end{array}$ & \multicolumn{2}{l}{ Groups of foreigners by citizenship in 2005} \\
\hline 1976 & 12154 & 0.3 & Europe & 80007 \\
1980 & 12502 & 0.3 & EU-countries & 42462 \\
1985 & 16478 & 0.3 & of which Sweden & 8265 \\
1990 & 26255 & 0.5 & of which Estonia & 17599 \\
1995 & 68566 & 1.3 & Russia & 25326 \\
2000 & 91074 & 1.4 & Other Europe & 12219 \\
2002 & 103682 & 1.6 & Africa & 11448 \\
2004 & 108346 & 2.1 & of which Somalia & 4623 \\
2005 & 113852 & 2.2 & America & 4641 \\
2006 & 121739 & 2.3 & Asia and Oceania & 24284 \\
& & & Unknown/without citizenship & 1359 \\
\hline
\end{tabular}

Economically active population aged $15-64$ by industry (\%)

\begin{tabular}{lrrrrrrr}
\hline & 1950 & 1960 & 1970 & 1980 & 1990 & 2000 & 2004 \\
\hline Agriculture and forestry, fishing & 45.8 & 35.5 & 20.3 & 12.6 & 8.5 & 6.1 & 4.2 \\
and hunting & 20.8 & 21.6 & 25.9 & 26.3 & 21.9 & 21.2 & 19.1 \\
Manufacturing, etc. & 6.3 & 8.7 & 8.3 & 7.1 & 7.0 & 6.4 & 6.1 \\
Construction & 9.5 & 13.6 & 18.9 & 19.1 & 26.3 & 27.5 & 29.1 \\
Trade, etc. & 5.4 & 6.3 & 7.1 & 7.9 & 7.2 & 7.4 & 7.5 \\
Transport and communications & 10.8 & 14.0 & 18.1 & 24.8 & 26.8 & 31.3 & 32.4 \\
Services & 1.4 & 0.3 & 1.4 & 2.2 & 2.3 & 0.3 & 1.7 \\
Unknown & 1960 & 2033 & 2118 & 2222 & 2332 & 2335 & 2262 \\
\hline 1000 persons & &
\end{tabular}

${ }^{1}$ Employed labor force. The grounds for classification have been changed in some extent in 1989 and in 1995. 
Females in labor force

\begin{tabular}{lcccc}
\hline & $\begin{array}{c}\text { The proportion of females } \\
\text { in labor force }(\%)\end{array}$ & \multicolumn{4}{c}{$\begin{array}{l}\text { Female labor force as a percentage of female population } \\
\text { aged } \\
20-44 \text { yrs }\end{array}$} & $15-64$ yrs & $15-74$ yrs \\
\hline $1960-64$ & 44.0 &.. &.. & 56.2 \\
$1965-69$ & 43.3 &.. &.. & 55.1 \\
$1970-74$ & 44.7 &.. &.. & 57.9 \\
$1975-79$ & 46.3 & 78.0 & 66.8 & 59.9 \\
$1980-841$ & 47.3 & 82.5 & 71.2 & 63.0 \\
$1985-891$ & 47.7 & 84.2 & 72.8 & 64.4 \\
$1990-94$ & 47.5 & 80.6 & 70.8 & 62.2 \\
$1995-99$ & 47.5 & 79.0 & 70.4 & 61.6 \\
2000 & 47.9 & 79.9 & 72.0 & 63.2 \\
2003 & 48.0 & 80.0 & 72.1 & 63.5 \\
2005 & 48.3 & 79.7 & 72.8 & 64.1 \\
2006 & 48.4 & 80.1 & 73.2 & 64.6 \\
2007 & 48.4 & 80.7 & 73.8 & 65.1 \\
\hline
\end{tabular}

${ }^{1}$ The data for 1981-87 have been corrected to correspond to the data of the revised Labor Force Survey from 1987 on.

\section{Unemployment rate}

\begin{tabular}{lccccc}
\hline & Both sexes & Men & $\begin{array}{c}\text { Men aged } \\
15-24\end{array}$ & Women & $\begin{array}{c}\text { Women aged } \\
15-24\end{array}$ \\
\hline $1965-69$ & 2.5 & 3.4 &.. & 1.3 &.. \\
$1970-74$ & 2.3 & 2.6 & 4.7 & 1.8 & 3.8 \\
$1975-79$ & 5.1 & 5.8 & 11.8 & 4.3 & 9.1 \\
$1980-84$ & 5.1 & 5.3 & 10.4 & 4.9 & 9.9 \\
$1985-89$ & 4.7 & 5.2 & 9.7 & 4.2 & 7.9 \\
$1990-94$ & 12.1 & 13.7 & 25.8 & 10.3 & 19.3 \\
$1995-991$ & 13.9 & 13.7 & 25.8 & 14.2 & 25.2 \\
2000 & 9.8 & 9.1 & 21.1 & 10.6 & 21.6 \\
2003 & 9.0 & 9.2 & 21.9 & 8.9 & 21.6 \\
2005 & 8.4 & 8.2 & 20.6 & 8.6 & 19.3 \\
2006 & 7.7 & 7.4 & 19.4 & 8.1 & 18.3 \\
2007 & 6.9 & 6.5 & & 7.2 & \\
\hline
\end{tabular}

${ }^{1}$ The grounds for classification of unemployment have been changed somewhat in 1997. 